\title{
Localized Bone Augmentation and Implant Site Development-Review with a case
}

Koirala $\mathrm{PK}^{1}$, Pradhan $\mathrm{S}^{2}$, Gorkhali $\mathrm{RS}^{3}$

${ }^{1}$ Senior Consultant, Department of Periodontology and Oral Implantology, National Academy of Medical Sciences, Bir Hospital, Kathmandu

${ }^{2}$ Professor, Department of Periodontology and Oral Implantology, National Academy of Medical Sciences, Bir Hospital, Kathmandu

${ }^{3}$ Assistant Professor, Department of Periodontology and Oral Implantology, National Academy of Medical Sciences, Bir Hospital, Kathmandu

\begin{abstract}
Guided bone regeneration (GBR) has been used for the regeneration of bone in conjunction with the placement of dental implants, for augmentation of resorbed alveolar crests, and to treat localized ridge deformities. It is based on the principle of protecting bone regeneration against overgrowth of tissues formed by rapidly proliferating non-osteogenic cells. In this case, the space created by the Titanium mesh supported platelet rich fibrin membrane was filled by tissues with features of newly formed bone. No residual bone defects were observed and an increase of the alveolar width and height was observed. No untoward effects on bone regeneration were observed except membrane exposure after 4 and $1 / 2$ months. This case shows a satisfactory result concerning GBR technique or implant site development.
\end{abstract}

Key words: Guided bone regeneration, Titanium mesh, Platelet rich fibrin membrane

\section{Introduction}

$\mathrm{B}$ ased on fundamental experimental studies performed by the research teams of Per Ingvar Branemark from the University of Gothenburg (Sweden) and Andre Schroeder from the University of Bern (Switzerland), the use of dental implants has become a scientifically accepted treatment for the replacement of lost or missing teeth in fully and partially edentulous patients. In landmark papers published in the late 1960s and 1970s, both research teams described the phenomenon of osseointegrated titanium implants. ${ }^{1,2}$ An osseointegrated implant is characterized by direct apposition of

\section{*Corresponding Author}

Dr. Pramod Kumar Koirala

Senior Consultant

Deparetment of Periodontology and Oral

Implantology

National Academy of Medical Sciences, Bir Hospital, Kathmandu

Email:drpkkoirala@gmail.com living bone to the titanium surface. ${ }^{3}$ To achieve osseointegration, the implant must be inserted with a low-trauma surgical technique to avoid overheating of the bone during preparation of a precise recipient site, and the implant should be placed with sufficient primary stability. ${ }^{4}$ When these clinical guidelines are followed, successful osseointegration will predictably occur as demonstrated in comparative, experimental studies. $^{5}$

Bone grafts and bone substitute materials play important roles in GBR procedures, with the indications ranging from minor implant fenestration defects to bridge major continuity defects in the facial skeleton. Guided bone regeneration and peri-implant contour augmentation are well established and inseparably connected to successful clinical implant dentistry. It has particular importance and therefore has been strongly promoted for prosthetically driven implant placement, rather than older bone-driven approach. 
GBR, a well-established procedure based on the principle of protecting bone regeneration

against overgrowth of tissues formed by rapidly proliferating nonosteogenic cells.

Autografts are the only grafting materials with well-documented osteoinductive potential. Like autografts, allografts contain osteoinductive molecules. Xenografts are, today, mainly used in the form of DBBM. The production process preserves the original cancellous geometry and natural surface characteristics, but the organic material is removed to exclude the risk of disease transmission. Non-crosslinked collagen membranes are currently the membrane of choice for all horizontal GBR and all sinus floor elevation as they have favorable clinical handling characteristics during surgery, including their hydrophilic quality.

Bone has the unique capability to rebuild its original structure and function in response to a defect or fracture. The pattern of bone healing closely resembles that of development and growth. Under stable mechanical conditions, bone is formed directly or primarily, provided that two essential conditions are present: an ample blood supply and a solid base for bone deposition. The solid base is provided by the surface of the fragment ends or by the bony margins of a defect.

Repair of bone defects closely resembles appositional growth. In the initial, rapid phase, a template of woven bone is constructed and gradually reinforced by lamellar bone. Depending on the local environment, this primary sponge work is then transformed into compact cortical or trabecular bone. In the second phase, the regenerate undergoes profound remodeling and substitution by bone remodeling units (bone metabolizing units). In cortical bone, the remodeling units produce secondary osteons.

Titanium implants is inserted in cases with alveolar crest defects, and the defects will be covered with platelet rich fibrin (PRF) membrane supported by a titanium mesh. PRF membrane is used in place of collagen membrane. After healing, the titanium mesh is removed.

\section{Case Report}

A 29-year-old female was referred for the replacement of upper left lateral incisor with dental implant. The root canal treated lateral incisors was intruded in an accident 3 months back which was extracted and replaced by removable partial denture. The patient opted for prosthetic rehabilitation with implant-supported fixed prosthesis and anatomical rehabilitation of the alveolar defect. Surgical procedures including basic surgical phases for implant placement and uncovering and bone graft was planned for the patient.

Hematological, biochemical and serological investigations were with in normal limits. Clinical examination showed a buccal-lingual horizontal alveolar defect with sufficient keratinized gingiva at the residual site. CBCT revealed available bone of over $5 \mathrm{~mm}$ width with a facial undercut. After the defect and remnant tissues were scaled and evaluated, treatment protocol was discussed with the patient. The buccal depression was about $5 \mathrm{~mm}$ in width, $4 \mathrm{~mm}$ horizontally and $6 \mathrm{~mm}$ vertically, which implied a bucket-shaped bony deficiency lacking more than half of the bucket wall. As it was difficult to maintain a stable space for ideal guided bone regeneration (GBR), this deficiency was preferred to be restored through block bone grafting or Titanium mesh. The residual crest bone was measured about $5 \mathrm{~mm}$ horizontally which seemed satisfactory for the placement of implant in optimal position. However, extremely thin buccal bony wall and high risk of implant exposure required GBR in this case. Insufficient residual bone for simultaneous implant placement may involve 
GBR alone for primary augmentation, with immediate or delayed implant placement which was explained to the patient.

A crestal incision and vertical relieving incision were made to raise a full mucoperiosteal flap. Implant was $3.3 \mathrm{~mm}$ in diameter and $11.5 \mathrm{~mm}$ in length. The surgical guide was positioned, and implant bed preparation was carried out using the implant drills. The lack of bone width became clearly evident; however, implant position was not compromised. The implant was placed with good primary stability. Cover screw was placed for primary closure.

Subsequently, the titanium mesh of measured size was fixed to the recipient site with a screw. To attain a natural contour, space was packed with Bio-Oss spongiosa granules of 0.25-1 mm (Geistlich Pharma, Wolhusen, Switzerland). Platelet rich membrane was prepared and placed over the mesh. Below the labial full-thickness flap, the split flap was repositioned apically to cover the titanium mesh and the particle substitutes. Flap closure was free of tension with interrupted sutures.

Four months after the procedure, ridge development with improved keratinized tissue was noted, and well osseointegrated implant was noticed in periapical radiograph. The titanium mesh exposure was observed in 4 and $1 / 2$ months. Then the titanium mesh was removed and the flap closure was done for maturation of graft particles. Labial soft tissue defect was managed by flap rolling technique. Suturing of the reflected thick tissue was done in such a way that it helped in augmentation of soft tissue. Following 4 weeks of soft tissue healing for the development of gingival cuffs, a traditional prosthetic procedure was followed. The implant-supported crown was fabricated and screwed on the abutment. The patient was satisfied with both esthetic and functional outcome.

\section{Discussion}

GBR can be used in conjunction with alloplast, allograft, or xenograft particulates as alternatives to autogenous bone. However, these matrices are often associated with limited or poor bone formation. On the other hand, in non-spacemaintaining defects, block grafts may exhibit significant advantages over particulate grafts with regard to contour reconstruction and resistance to micromovement. In this case, harvesting of bone adjacent to the defect avoided additional trauma to the donor site. Moreover, transplant of the autogenous bone block resulted in an intrabony-like space with sufficient exposure of the bone marrow, which was suitable for filling and maintaining particulate bone substitutes and provided better vascularization for bone regeneration. ${ }^{7}$

In compromised clinical situations, it is recommended that implants be placed several months after bone grafting because their placement at the time of tissue grafting is appropriate only when optimal positioning can be achieved. ${ }^{8}$

A recent systemic review concluded that survival rates of implants placed into augmented areas were comparable with that of implants placed into pristine bone. ${ }^{9}$ However, identifying best grafting technique for alveolar ridge augmentation remains challenging. Most studies of lateral or vertical ridge augmentation have reported on the findings of two-stage approaches, exposure of a second surgical site to harvest autogenous graft material, ridge splitting/expansion techniques, or alveolar distraction osteogenesis that were associated with higher morbidity and increased treatment time. ${ }^{10}$ Thus single-stage implant placement technique with simultaneous grafting to support the soft-tissue margin approximately $1 \mathrm{~mm}$ above the implant margin was done in this case. 


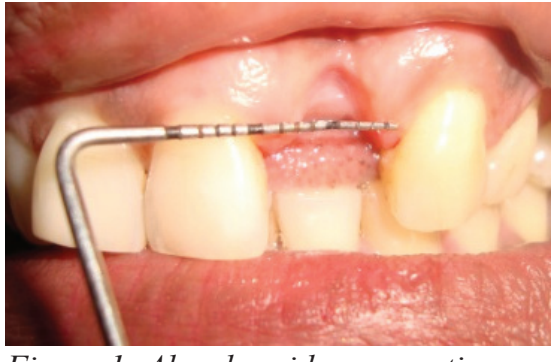

Figure 1: Alveolar ridge resorption

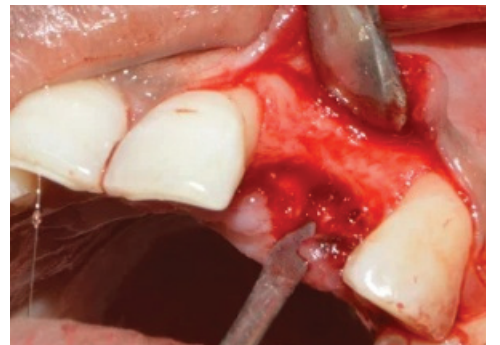

Figure 2: Ridge width in the crestal area

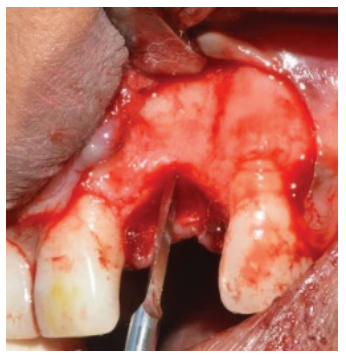

Figure 3: Trapezoidal flap design

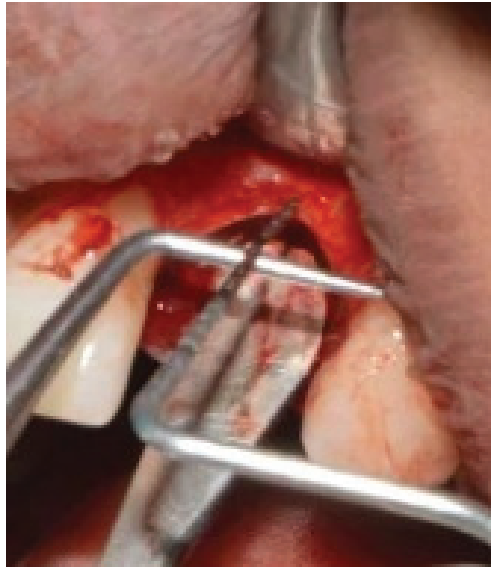

Figure 4: Vertical ridge level measured

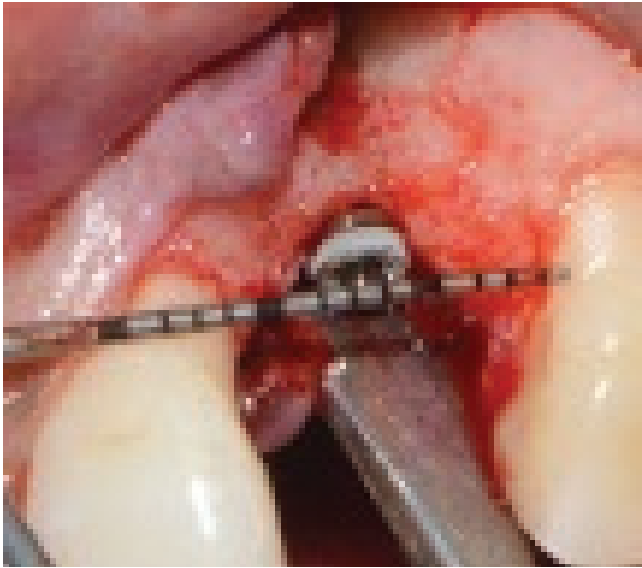

Figure 5: implant placed

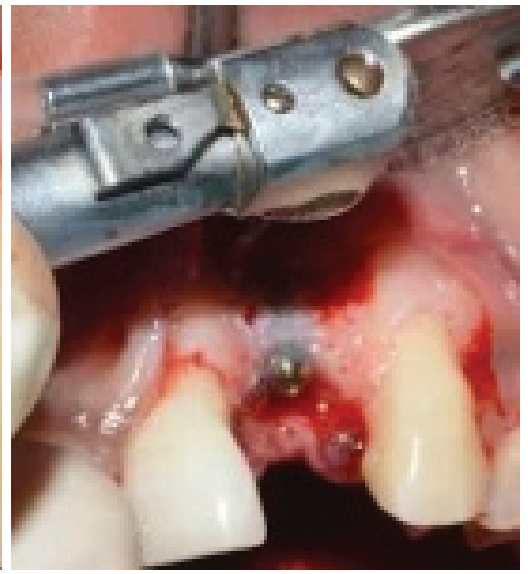

Figure 6: Numerous drill holes peri-implant cortex
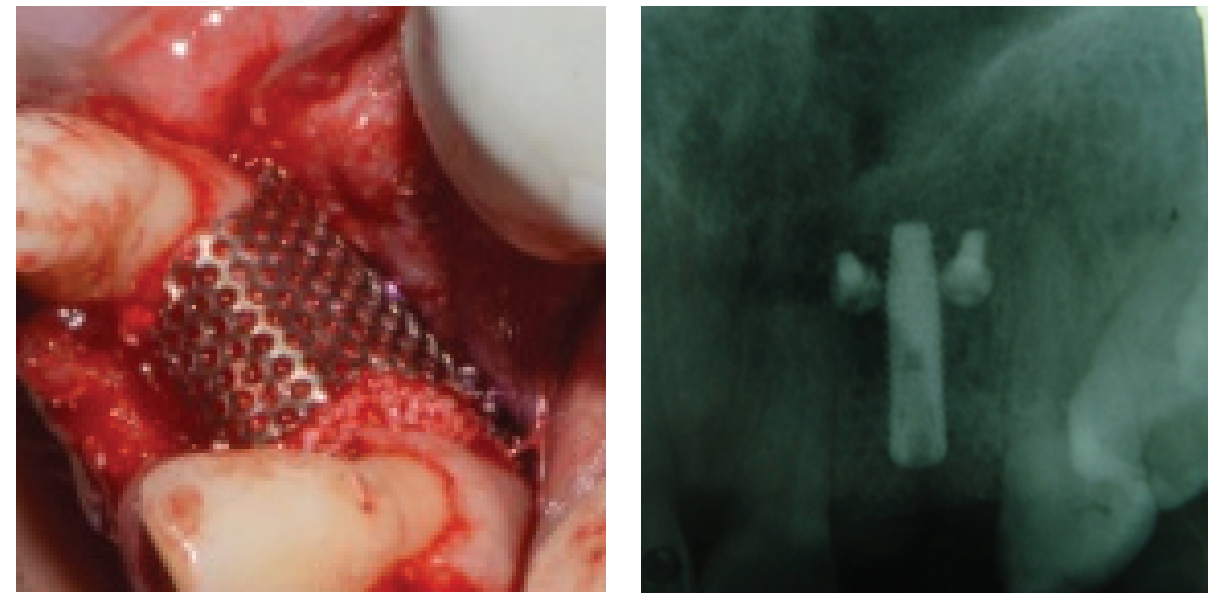

Figure 7: Titanium mesh tucked over Bio Figure 8: Radiograph of graft site oss at defect site with $1.5 \mathrm{~mm}$ Ti screw

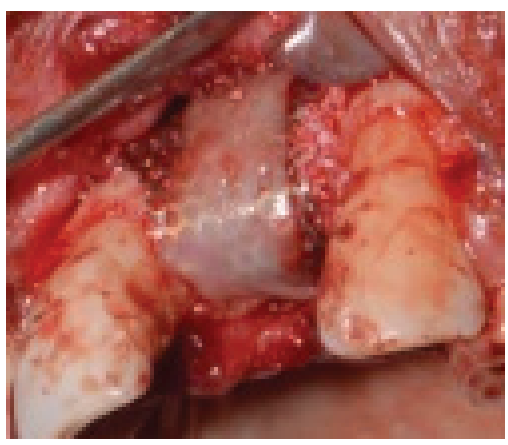

Figure 9: PRF membrane placed over titanium mesh

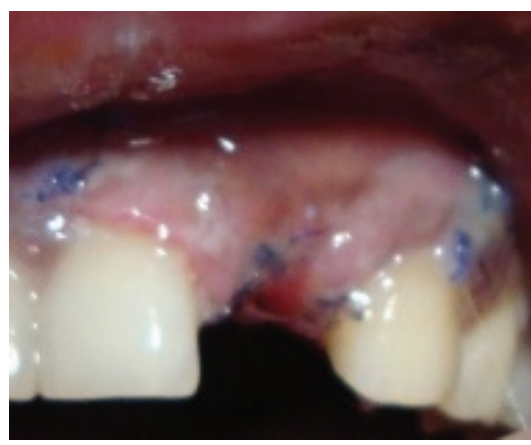

Figure 10: After 1 week of closure of flap

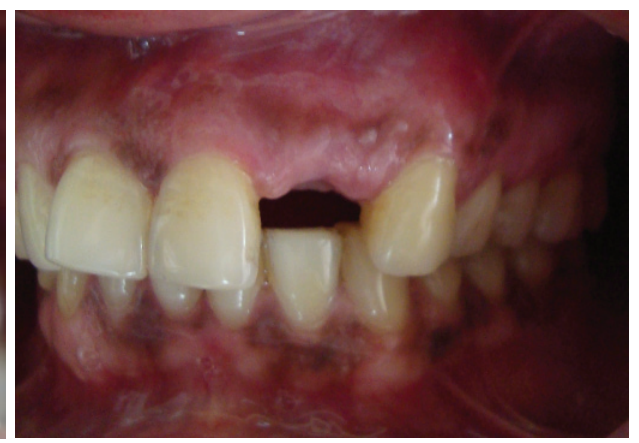

Figure 11: Osseointegration phase operation site 


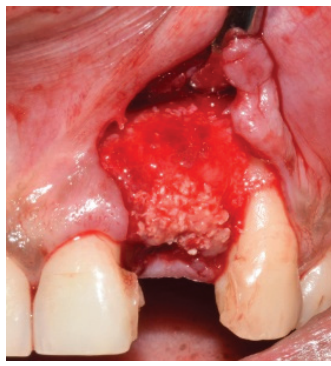

Figure 12: After $4 \& 1 / 2$ month of Titanium

mesh removal

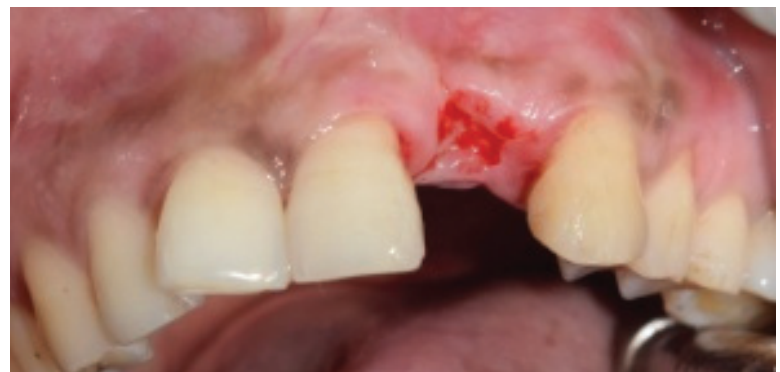

Figure 13,14. Soft tissue de-epithelization before flap reflection

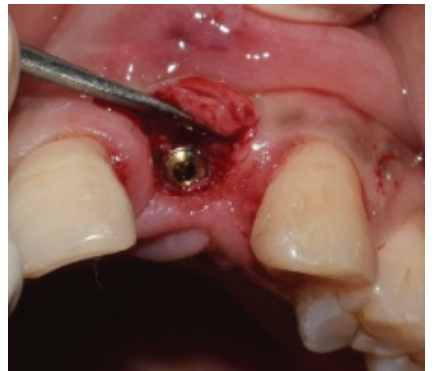

Rolled flap

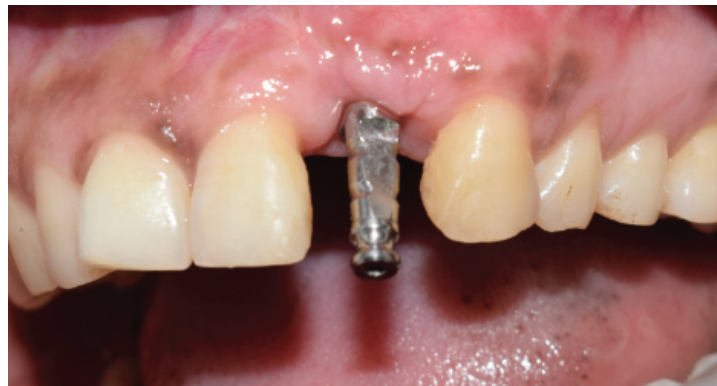

Figure 15: Impression coping

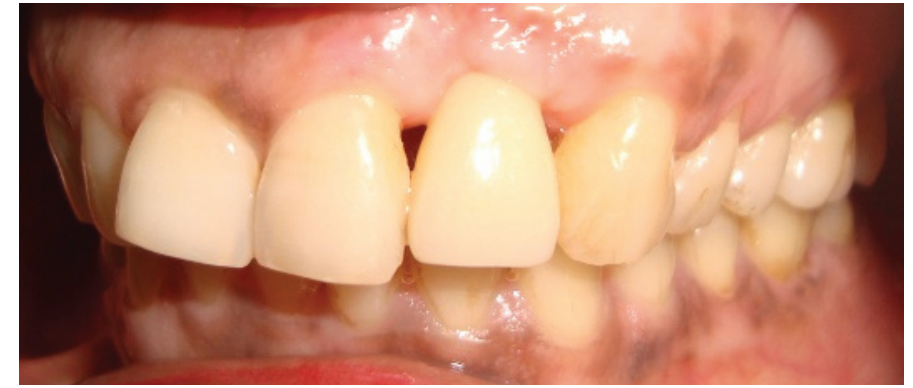

Figure 16: Final prosthesis

\section{Conclusion}

Single-stage implant placement and simultaneous grafting with GBR technique using mineralized particulate graft supported by titanium mesh and PRF membrane showed promising outcome in correcting small to medium sized vertical labial wall defects.

\section{References}

1. Branemark PI, Breine U, Adell R, Hansson B, Lindstrom J, Ohlsson A. Intra-osseous anchorage of dental prostheses: I. Experimental studies. Scand J Plastic Reconstructive Surg. 1969;3(2):81-100.

2. Schroeder A, van der Zypen E, Stich H, Sutter F. The reactions of bone, connective tissue, and epithelium to endosteal implants with titaniumsprayed surfaces. J Maxillofac Surg. 1981;9:1525.

3. Albrektsson T, Branemark PI, Hansson HA, Lindstrom J. Osseointegrated titanium implants: requirements for ensuring a long-lasting, direct bone-to-implant anchorage in man. Acta Orthopaedica Scandinavica. 1981;52(2):15570 .
4. Buser D, Von Arx T, Ten Bruggenkate C, Weingart D. Basic surgical principles with ITI implants Note. Clin Oral Implants Res: Chapter 3. 2000;11:59-68.

5. Weber H, Buser D, Donath K, Fiorellini J, Doppalapudi V, Paquette D, et al. Comparison of healed tissues adjacent to submerged and non-submerged unloaded titanium dental implants. A histometric study in beagle dogs. Clin Oral Implants Res. 1996;7(1):11-9.

6. Lindfors LT, Tervonen EA, Sándor GK, Ylikontiola LP. Guided bone regeneration using a titanium-reinforced ePTFE membrane and particulate autogenous bone: the effect of smoking and membrane exposure. Oral Surg Oral Med Oral Patho Oral Radio Endodontol. 2010;109(6):825-30.

7. Lacerda SA, Lanzoni JFA, Bombonato-Prado KF, Campos AA, Prata CA, Brentegani LG. Osteogenic potential of autogenous bone associated with bone marrow osteoblastic cells in bony defects: a histomorphometric study. Implant Dentistry. 2009;18(6):521-9.

8. Mills MP. Comparative Study of Buccal Dehiscence Defects in Immediate, Delayed, and Late Maxillary Implant Placement with 
Collagen Membranes: Clinical Healing Between Placement and Second Stage Surgery. Implant Dentistry. 2003;12(3):200.

9. Klein MO, Al-Nawas B. For which clinical indications in dental implantology is the use of bone substitute materials scientifically substantiated? European J Oral Implantol. 2010;4:11-29.
10. Chiapasco M, Tommasato G, Palombo D, Scarnò D, Zaniboni M, del Fabbro M. Dental implants placed in severely atrophic jaws reconstructed with autogenous calvarium, bovine bone mineral, and collagen membranes: A 3-to 19-year retrospective follow-up study. Clin Oral Implants Res. 2018;29(7):725-40. 